\title{
Antibiotic resistance among Shigella serogroups isolated in Tehran, Iran (2002-2004)
}

\author{
Reza Ranjbar ${ }^{1}$, Mohammad Mahdi Soltan- Dallal ${ }^{2}$, Mohammad Reza Pourshafie ${ }^{3}$, Caterina \\ Mammina ${ }^{4}$ \\ ${ }^{1}$ Molecular Biology Research Center, Baqiyatallah University of Medical Sciences, Tehran, Iran \\ ${ }^{2}$ Department of Pathobiology, School of Public Health and Institute of Public Health Research, Tehran University of Medical \\ Sciences, Iran \\ ${ }^{3}$ Department of Microbiology, Institute Pasteur of Iran, Tehran, Iran \\ ${ }^{4}$ Department of Sciences for Health Promotion “G. D'Alessandro”, University of Palermo, Via del Vespro 133, I-90127 Palermo, \\ Italy
}

Key words: Shigella, antibiotic resistance, Iran

J Infect Dev Ctries 2009; 3(8):647-648.

Received 22 September 2008 - Accepted 12 August 2009

Copyright ( 2009 Ranjbar et al. This is an open-access article distributed under the Creative Commons Attribution License, which permits unrestricted use, distribution, and reproduction in any medium, provided the original work is properly cited.

Shigellosis, one of the most common bacterial diarrhoeal diseases, is endemic throughout the world. It is one of the major causes of morbidity in children with diarrhoea in Iran [1-4].

Antibiotic therapy is useful and effective for shigellosis. Treatment is critical for persons with severe disease, particularly children and immunosuppressed patients. Use of an appropriate antibiotic therapy can shorten the duration of symptoms, significantly reduce the risk of transmission, and also prevent potentially lethal complications [5-7]. However, high frequencies of resistance in Shigella spp. to commonly used antimicrobial agents have been reported in recent years worldwide $[8,9]$.

The aim of this study was to investigate the current antimicrobial resistance trends among the strains of Shigella recovered from children with acute diarrhoea in Tehran, Iran, during the years 2002-2004.

Of a total of 200 Shigella spp. isolates studied, $110,84,5$, and one were identified as $S$. sonnei, $S$. flexneri, S. boydii, and $S$. dysenteriae, respectively. These strains were isolated from pediatric patients (younger than 12 years old) with acute diarrhoea admitted to five major hospitals in Tehran, Iran. Antimicrobial susceptibility testing was performed according to the standard guidelines of the Clinical and Laboratory Standards Institute [10].
Overall, more than $97.5 \%$ of tested isolates were resistant to streptomycin, $93.5 \%$ to sulfamethoxazoletrimethoprim, $93.5 \%$ to tetracycline, $8.0 \%$ to kanamycin, $1.5 \%$ to cefixime and $0.5 \%$ to amikacin. Most strains of S. flexneri (95.2\%) were resistant to amoxicillin-clavulanic, while strains belonging to other serogroups were fully susceptible to this antibiotic. None of the strains of $S$. flexneri and $S$. dysenteriae and one strain only of $S$. boydii was resistant to nalidixic acid, but nine $(8.2 \%)$ isolates of $S$. sonnei were resistant to this antibiotic. More than $72.6 \%$ of $S$. flexneri isolates, but $2.7 \%$ only of $S$. sonnei isolates, were resistant to ampicillin. More than $32.1 \%$ of $S$. flexneri strains were resistant to chloramphenicol, while only one isolate of $S$. boydii was resistant to this antibiotic.

None of the tested isolates was resistant to cephalothin, cefotaxime, ceftriaxone, ceftizoxime, ceftazidime, gentamicin or ciprofloxacin.

The results indicate that most Shigella isolates show high resistance rates to streptomycin, sulfamethoxazole-trimethoprim and tetracycline. When compared to previous reports from Iran, an increase in the prevalence of resistance to tetracycline and sulfamethoxazole-trimethoprim is evident. Previously, resistance to tetracycline was reported at $77 \%, 56 \%$, and $74 \%$ by different authors in 1984 , 1989 and 2001 [11-13]. Similarly, 94\% of our isolates were found to be resistant to 
sulfamethoxazole-trimethoprim in comparison with a previously reported $70 \%$ prevalence [13]. It can be concluded that these antimicrobial agents are no longer appropriate for empirical therapy of shigellosis in our country.

On the other hand, the resistance rate proves to be consistently low for ampicillin, cefixime, amikacin, kanamycin and chloramphenicol. Among the different serogroups, only S. flexneri isolates $(32.1 \%)$ were resistant to chloramphenicol, a finding which is consistent with that of a previous study conducted in North America [14]. This is an interesting observation because $S$. flexneri is no longer the predominant species in Iran as it was in the past [1,2]. A cause of concern is the increasing frequency of resistance to nalidixic acid in $S$. sonnei, which has increased to $5 \%$ from a previous reported prevalence of approximately $1 \%$ in 2001 [13]. It is also noteworthy that none of our Shigella strains was resistant to gentamicin, ciprofloxacin, and thirdgeneration cephalosporins.

In conclusion, we showed the prevalence of resistance to some commonly used antibiotics is high and differs within Shigella serogroups. While antibiotic resistance varies by Shigella serogroups, continuous monitoring of resistance patterns among individual serogroups is recommended. Our findings emphasize the need to reassess the use of antibiotic agents according to Shigella serogroup in the treatment of shigellosis in our country.

\section{Acknowledgements}

The authors are thankful for the cooperation of Dr. Mohammad Taghi Ashtiani and Mrs. Mina Abedini from the Microbiology laboratory of the Children's Medical Center. We would also like to thank Dr. Mohammad Rahbar from Millad Hospital.

\section{References}

1. Ranjbar R, Aleo A, Giammanco GM, Dionisi AM, Sadeghifard N, Mammina C (2007) Genetic relatedness among isolates of Shigella sonnei carrying class 2 integrons in Tehran, Iran, 2002-2003. BMC Infect Dis 22: 62.

2. Ranjbar R, Mammina C, Pourshafie MR, Soltan-Dallal MM (2008) Characterization of endemic Shigella boydii strains isolated in Iran by serotyping, antimicrobial resistance, plasmid profile, ribotyping and pulsed-field gel electrophoresis. BMC Res Notes 1: 74.

3. Ranjbar R, Soltan Dallal MM, Talebi M, Pourshafie MR. (2008) Increased isolation and characterization of Shigella sonnei obtained from hospitalized children in Tehran, Iran. J Health Popul Nutr 26: 426-430.
4. Hosseini MJ, Ranjbar R, Ghasemi H, Jalalian HR. (2007) The prevalence and antibiotic resistance of Shigella spp. recovered from patients admitted to Bouali hospital, Tehran, Iran during 1999-2000. Pak J Biol Sci 10: 2778-80.

5. DuPont HL. Shigella species (bacillary dysentery). In: Mandell GL, Bennett JE, Dolin R, eds. Mandell, Douglas and Bennett's principles and practice of infectious diseases. 5th ed. Vol 2. New York: Churchill Livingstone, 2000: 2363-9.

6. Voogd CE, Schot CS, Van Leeuwen WJ, and Van Klingeren B (1992) Monitoring of antibiotic resistance in Shigella isolated in the Netherlands 1984-1989. Eur J Clin Microbiol Infect Dis 11: 164-167.

7. Salam M and Bennish ML (1991) Antimicrobial therapy for shigellosis. Res Infect Dis 13 (Suppl 4): S332-S341.

8. Bennish ML, Salam MA, Hossain MA, Myaux J, Khan EH, Chakraborty J (1992) Antimicrobial resistance of Shigella isolates in Bangladesh, 1983-1990: increasing frequency of strains multiply resistant to ampicillin, trimethoprimsulfamethoxazole, and nalidixic acid. Clin Infect Dis 14: 1055-1060.

9. Fullá N, Prado V, Durán C, Lagos R, Levine MM. (2005) Surveillance for antimicrobial resistance profiles among Shigella species isolated from a semirural community in the northern administrative area of Santiago, Chile. Am J Trop Med Hyg 72: 851-4.

10. Clinical and Laboratory Standards Institute. Performance standards for antimicrobial susceptibility testing; fifteenth informational supplement. Approved standard M100-S15. Wayne, PA: Clinical and Laboratory Standards Institute, 2005.

11. Nikkah J and Mehr-Movahead A (1988) Antibiotic resistance among Shigella species isolated in Tehran, Iran. Ann Trop Med Parasitol 82: 481-3.

12. Katouli M, Pachenary A, Jaafari A, Asghar A, Moghaddam F, Dehaghi NH et al. (1989) The role of Shigella spp. in childhood diarrhoea in Iran and their antibiotic resistance. Scand J Infect Dis 21: 415-9.

13. MoezArdalan K, Zali MR, Dallal MM, Hemami MR, Salmanzadeh-Ahrabi S. (2003)Prevalence and pattern of antimicrobial resistance of Shigella species among patients with acute diarrhoea in Karaj, Tehran, Iran. J Health Popul Nutr 21: 96-102.

14. Sivapalasingam S, Nelson JM, Joyce K, Hoekstra M, Angulo FJ, Mintz ED (2006) High prevalence of antimicrobial resistance among Shigella isolates in the United States tested by the National Antimicrobial Resistance Monitoring System from 1999 to 2002. Antimicrob Agents Chemother 5: 49-54.

\section{Corresponding author}

Reza Ranjbar

Molecular Biology Research Center

Baqiyatallah University of Medical Sciences

Tehran, Iran

Tel \& Fax: +98-21-88039883

Email: ranjbar@bmsu.ac.ir

Conflict of interest: No conflict of interest is declared. 